\title{
Research on the Dispersant Effectiveness with the Action of Waves
}

\author{
Qiaomin Wang ${ }^{a}$, Bing Sun ${ }^{b,}$, Zhiyu Yan ${ }^{c}$, Mi Wend, Hui Liu ${ }^{e}$, Ying $\mathrm{Yu}^{\mathrm{f}}$ \\ Environmental Science and Engineering College, Dalian Maritime University, Dalian 116026, China

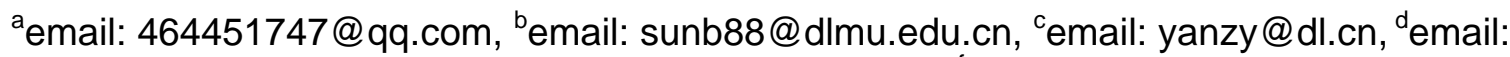

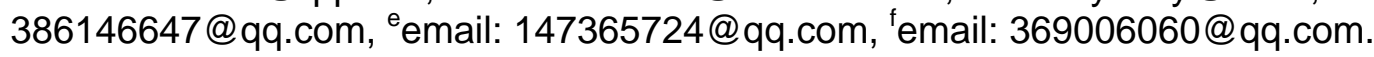

*Corresponding author

Keywords: Dispersant, Effectiveness, Waves.

\begin{abstract}
Oil spill dispersant, as a kind of effective measures to deal with oil spill, has been widely used, but the effectiveness still needs more investigations. In this paper, what factors influenced the dispersant effectiveness was studied in the tank with the action of waves of different time intervals, and dispersant relative content in oil slick and oil concentration in seawater were mainly the two aspects to examine the phenomenon of dispersant away from oil and dispersion of oil spill. The results showed that the degree of dispersant away from oil increased over with the wave action time, but oil dispersion had not obvious regulation with it. It was speculated that, in actual seawater body waves prompted dispersant to be away from oil, but can also maintained the dispersion effectiveness. This research provided certain technical support for comprehensively and objectively evaluating the dispersant effectiveness.
\end{abstract}

\section{Introduction}

With the rapid development of oil exploitation and transportation, oil spill accidents had frequently occurred, which made marine creatures and natural resources severely damage. Once oil spilled into the sea, it would undergo natural dispersion, and form oil-in-water droplets with the action of waves. The oil droplets would eventually be diluted to lower than the threshold toxicity [1-4]. Oil spill dispersant, as a kind of effective measures to deal with oil spill, could greatly enhance oil film dispersing into water body, because it can effectively reduce interfacial tension between oil and seawater [5]. In recent years, with the research and development of low toxicity and high efficient dispersant, the negative impact of oil spill in marine environment was immensely weakened. Except for convenient operation, the dispersant weaken the damage to aquatic animals and reduced the oil pollution to coastline environmental to a great extent [6].

Dispersion of oil was affected by the chemical properties of oil and dispersant, and various environmental factors [7]. Mixing energy was the premise of dispersed oil droplets into seawater, and emulsification oil required more mixing energy to stay in water body. Simulation studies in the laboratory showed that [8] the size of oil droplets was negative correlation to how much the mixing energy inputted to the test container. Namely, the larger the mixing energy was, the smaller oil droplets was. Some standardized bench tests were often used to assess the effectiveness of specific chemical dispersants, so as to provide reference data for choosing appropriate dispersant [9-12]. However, compared with the actual sea environment these tests were limited, mainly because of failing to consider conveying and dilution effect in the water body [6], which had effect on gather again of dispersed oil. During the period of physical and chemical dispersion of oil spill, turbulent structure in the water body was significantly important to vertical transmission, and also affected oil slick to disintegrate and dive. But at a certain depth that turbulent not reached, dispersion stability may be affected, and the recent tests failed to reflect the characteristics above. In order to overcome the constraints above, experimental methods and facilities needed to be improved according to the characteristics of mixing energy, and built an evaluation system of the chemical dispersant effectiveness that was closer to the actual situation.

Oil spill was composed of floating oil and oil droplets in the water body. So the dispersant effectiveness was investigated from two aspects above. The dispersant effectiveness was researched 
in wave tank with the action of intermittent waves in this paper. The influence of mixing energy on dispersion was studied by changing the wave action time, and dispersion dynamic on the seawater surface with broken waves and underwater waves not reached were examined through repeated waving and standing, which both provided a new thinking for mechanism research and effectiveness evaluation of dispersant, as well as the research and development of dispersant.

\section{Experiments}

Materials and Instruments. In this study, the research objects were Bohai crude oil and FuKen-2 dispersant. Test instruments were Shimadzu GC-2010 gas chromatograph (Shimadzu Corp., Kyoto, Japan) and T6 new century UV-vis spectrophotometer (Beijing Purkinje General Instrument Ltd., Beijing, China), respectively.

Methods. The mixture of dispersant and oil was fully stirred, added into seawater (artificial seawater, salinity was approximately $34 \%$ ), and then acted by waves in different intermittent wave patterns. The dispersant relative content and oil concentration were tested to investigate the separation characteristics and dispersion effectiveness of dispersant.

The dimensions of the tank was $49 \mathrm{~cm}$ (length)、31.5 cm (width) and $40 \mathrm{~cm}$ (height). Adding 23 $\mathrm{cm}$ deep artificial seawater into tank, which was about $35.5 \mathrm{~L}$. Adding dispersant into $20 \mathrm{ml}$ oil according to the volume proportion of that dispersant to oil was 0.4 (the ratio was in the dispersant using range of $20 \% \sim 70 \%$ ). Waves was produced by the plate beating the seawater that was into the underwater about $45 \mathrm{~mm}$. Beating frequency was about $98 \pm 2$ times/min, and the height of waves was about $30 \sim 40 \mathrm{~mm}$. There were 3 kinds of intermittent wave patterns, namely, waves lasted for 1,2 , and $3 \mathrm{~h}$, and then standing for $2 \mathrm{~h}$, after which waves again and standing again, so totaled four cycles. The difference among three patterns lied in the wave time per cycle. Wave pattern was expressed in Wi ( ' $i$ ' was the wave time in each cycle, and i=1, 2 and 3). W0 was the blank control for no wave action. Floating oil sampling time and experiment parameter were shown in Fig. 1. All experiments were completed under the room temperature of $23 \pm 2{ }^{\circ} \mathrm{C}$.

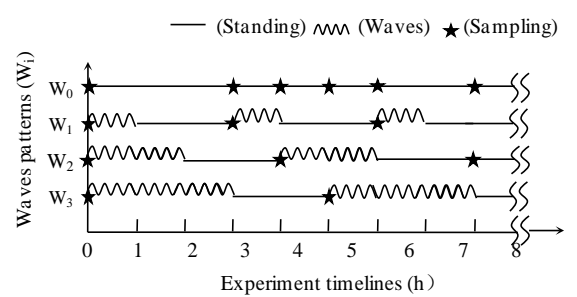

(a)

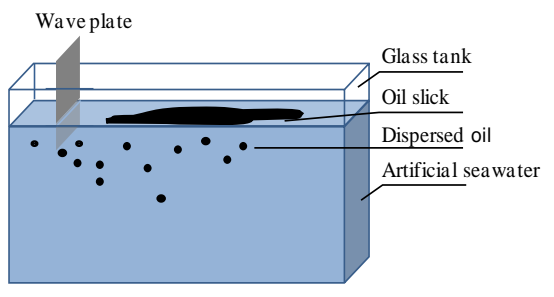

(b)

Fig.1: 4 kinds of intermittent wave patterns within $8 \mathrm{~h}$ (a) and the experiment schematic (b)

Sample Analysis Methods.Oil slick of $0.2 \mathrm{~g}$ was sampled to a beaker, and dissolved by $15 \mathrm{ml}$ organic solvents. First of all, chromatographic column (the chromatographic column from bottom to top was in turn filled with cotton wool, $5 \mathrm{~g}$ silica gel, and $1.2 \mathrm{~g}$ anhydrous sodium sulfate) was eluted by $10 \mathrm{ml}$-hexane. After elution, adding the dissolved oil sample into chromatographic column. Finally, the eluent was collected in a vitro for testing.

Seawater in the tank was sampled $20 \mathrm{ml}$ into the beaker, and extracted well by $20 \mathrm{ml}$ organic solvent in a separatory funnel. Extraction liquid was eluted through the chromatographic column above, and then diluted with organic solvent above to $25 \mathrm{ml}$ for testing.

GC Testing Conditions. The temperature of the inlet and the detector were respectively $280{ }^{\circ} \mathrm{C}$ and $300{ }^{\circ} \mathrm{C}$, and the carrier gas was high purity N2. Temperature program was that: the initial temperature was $60{ }^{\circ} \mathrm{C}$ at first, then increased to $100{ }^{\circ} \mathrm{C}$ by $20{ }^{\circ} \mathrm{C} / \mathrm{min}$, keeping for $2 \mathrm{~min}$; and then increased to $280{ }^{\circ} \mathrm{C}$ by $8{ }^{\circ} \mathrm{C} / \mathrm{min}$, keeping for $55 \mathrm{~min}$.

\section{Results and Discussion}

Dispersant Relative Content in Oil Slick.Mixed with oil firstly and then seawater were the premise of dispersant playing a role, mixing energy was provided by waves. The mixing function of 
waves was reported more in some studies, but whether the dispersant effectiveness was continuously influenced and dispersed oil was maintained by waves or not was also equally crucial. Dispersant relative content in oil slick changing with time was tested in this paper, aiming at investigating mixing characteristics and dispersion effectiveness between dispersant and oil under the alternating action of waves and standing. The results was shown in Fig. 2.

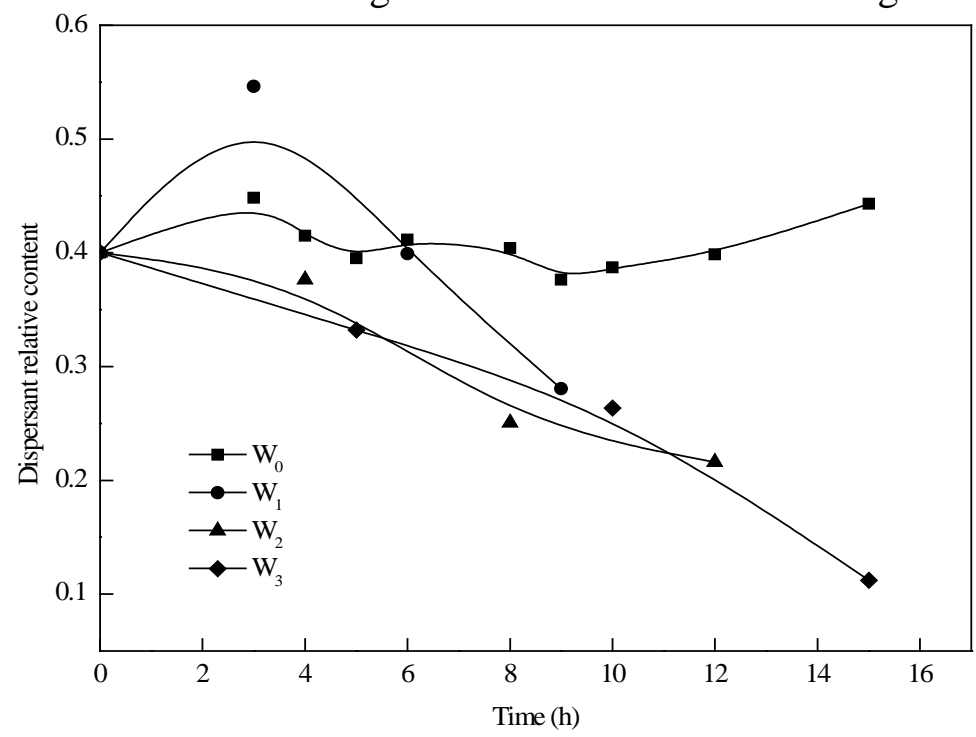

Fig.2: The curve of the dispersant relative content changing with time

As was shown in Fig.2, although the sample analysis value at $3 \mathrm{~h}$ was slightly high in no wave pattern (W0), dispersant relative content overall changed also slightly, and the process of repeated dispersing and floating was also not observed. Therefore, dispersant would not significantly away from the oil in the short term in W0. Sample analysis value at 2 h was about 0.55 in W1, which was significantly bigger than the initial value of 0.4 . The cause may be that emulsified and containing seawater oil slick included dispersant dissolved out of oil under the action of waves, which made oil content in samples was less, but the dispersant content was not less. Therefore, dispersant relative content increased, which was also appeared in the similar experiments.

Compared W1, W2 and W3, it was found that the longer the wave action time was, the greater the degree of dispersant away from oil was. From the initial to the end of the fourth cycle, dispersant relative content of W1, W2 and W3 dropped to 70\%, 54\% and 24\%, which has fallen $30 \%, 46 \%$ and $72 \%$.

The results showed that dispersant was rapidly away from oil during the process that oil repeatedly dispersing and floating, and the degree associated with wave action time. The longer the time was, the faster the dispersant relative content declined. The principal causes were that when dispersant mixed with oil contact with seawater, part of dispersant stayed in water body instead of floating with oil in the standing process. Because dispersant hydrophilic was stronger than hydrophilic. With the wave action time increased, the dispersant, oil and seawater mixed more fully, and more and smaller oil droplets dispersed into seawater, which was also observed in the experiments. Namely, oil droplets was smaller, dispersant was more easily to be away. At the same time, no wave pattern can simulate the actual seawater that waves not reached. It can speculate that dispersant would not be out of oil in that environment.

Oil Concentration in Seawater.After adding oil with dispersant into seawater, the oil would be dispersed under the action of intermittent waves, and then gathered and floated repeatedly. The oil concentration can reflect oil dispersion and sampled at $1 \mathrm{~h}, 3 \mathrm{~h}, 7 \mathrm{~h}$ and $13 \mathrm{~h}$. Test results were shown in Fig. 3. 


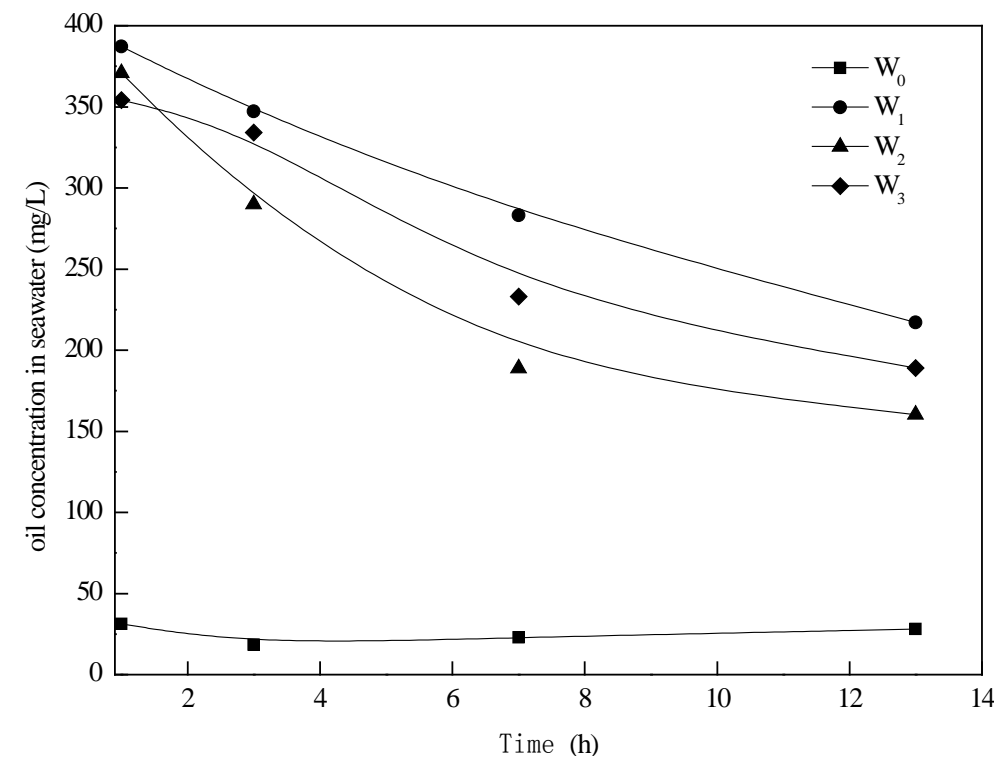

Fig.3 The curve of the oil concentration in seawater changing with time

As was shown in Fig.3, oil concentration declined with time down to 56\%, 56\% and 43\%, which fell by 44\%, 57\% and 44\%, in W1, W2 and W3 from the initial to the end of the experiment. This was mainly because the oil droplets in seawater gradually separated from dispersant, and then floated to the surface for gathering when the seawater was standing. In addition that dispersion ability of dispersant declined when it was from oil. The two above collectively resulted in oil concentration in seawater declined. From the previous analysis, the longer the wave action time was, the faster the dispersant was away from oil, therefore the greater the falling degree of dispersion effectiveness should also be. But it was found that part of oil reached the bottom that waves not got under the action of waves. The longer the wave action time was, the more dispersed oil was under the above situation, and the better the dispersion effectiveness was. Vertical distance was far, coupled with the wave action, leading to the oil floating speed was slow and less involved in the process of repeated dispersion and floatation. As a result, from this perspective, the longer the wave action was, the better the dispersion effectiveness was. So comprehensive results as follows: oil concentration with time decreased, but the falling degree had no obvious regularity with the wave action time.

In actual seawaters, a certain degree of wave action can enhance the dispersion of oil, making the dispersant better carry oil into seawater and also increasing dispersant into seawater from oil, which was evidenced by the results of Fig. 2 and Fig. 3. The longer the wave action time was, the greater the degree of dispersion was, and the more easily dispersant deviated from the floating oil. It was found that dispersed oil repeatedly dispersed, gathered and floated only in a certain depth range, which made the dispersant increasingly out of oil, and dispersion effectiveness decline. When wave action time increased, dispersed oil would be into deeper and wider seawater, and reach the bottom that waves not got. At this time, the underwater was similar to clam water body, and the activities of dispersed oil were weak. Therefore, long-range potentiation began to play the dominant role, and dispersed oil was not easy to gather and float under the joint action of dispersant and waves.

Waves not only prompted dispersant away from oil and decreased dispersant effectiveness, but also hindered dispersed oil floated and maintained it in seawater. The opposite tendency made dispersant effectiveness impacted by waves was more complex. Namely oil dispersion degree was related to the wave action time, and associated with seawater depths that dominated long-range potentiation. So there seems to be a key that making sense of the quantitative relationship between dispersant and these factors, which would help to further research dispersant action mechanism in the actual seawater, and also an important content that more comprehensively and objectively evaluating the dispersant effectiveness. 


\section{Conclusions}

In the proposed algorithm, the multi-objective bilevel programming problem is transformed into a single-level problem by using interpolation functions of the lower level solutions. The process avoids solving the lower level problems frequently, and reduces the computational cost. The major advantage of this algorithm is that it can solve some complex issues, in which the lower level problems are non-convex and non-differentiable. Hence, it can be used to deal with hard multiobjective bilevel programming problems.

The dispersant effectiveness was researched through the different intermittent wave actions in this study and the conclusions as following.

(1) The dispersant relative content was falling due to oil repeatedly dispersed and floated under the action of intermittent waves. The degree of dispersant away from oil increased with wave action time increased, and maximum reduction to $72 \%$.

(2) Dispersed oil decreased significantly over time, about 44\% 57\%. The reduction had no obvious regularity to wave action time. This is mainly because that the longer the wave action time was, the more easily dispersant was away from floating oil, but also the better dispersed oil reserved in seawater.

(3) It could be speculated that in the actual water body waves can accelerate the dispersant away from oil but also maintain dispersion effectiveness in the place where the waves not reached from the experimental results.

\section{Acknowledgements}

The research was financially supported by the National Natural Science Foundation of China (41 206095, 21207010), Ph.D. Programs Foundation of Ministry of Education of China (201221 25120010) and the Fundamental Research Funds for the Central Universities (No. 3132013091).

\section{References}

[1] K. Lee: submitted to Spill Science \& Technology Bulletin (2002).

[2] M. Li, C. Garrett: submitted to Marine Pollution Bulletin (1998).

[3] J.M. Shaw: submitted to Spill Science \& Technology Bulletin (2003).

[4] P. Tkalich, E.S. Chan: submitted to Marine Pollution Bulletin (2002).

[5] R.R. Lessard, G. Demarco: submitted to Spill Science \& Technology Bulletin (2000).

[6] NRC, National Research Council: Understanding Oil Spill Dispersants: Efficacy and Effects (National Academies Press, Washington 2005).

[7] M.F. Fingas: submitted to Surfactants: Fundamentals and Applications to the Petroleum Industry (2000).

[8] M.F. Fingas, D.A. Kyle, E.J. Tennyson, in The Sixteenth Arctic and Marine Oil Spill Program Technical Seminar. Gatineau, Quebec, Canada: Ministry of supply and service (1993).

[9] V.J. Kaku, M.C.B. M.ASCE, A.D. Venosa: submitted to Journal of environmental engineering (2006).

[10] D.M. White, I. Ask, C. Behr-Andres: submitted to Journal of Cold Regions Engineering (2002). [11] A. Moles, L. Holland, J. Short: submitted to Spill Science \& Technology Bulletin (2002).

[12] Z.K. Li, K. Lee, T. King, et al: submitted to Marine Pollution Bulletin (2008). 